\title{
Efecto de los parámetros fisicoquímicos y biológicos sobre la calidad del agua del río pisco
}

\section{Effect of physicochemical and biological parameters on the water quality of the pisco river}

\author{
DOI: $10.46932 / \mathrm{sfjdv2n4-049}$
}

Received in: March 1st, 2021

Accepted in: May 30th, 2021

\author{
Misael A. Bendezu Bendezu \\ Ingeniero Químico, Docente Asociado, Ica, Perú \\ Universidad Nacional San Luis Gonzaga de Ica, Urb. Las Dunas H-15, Ica, Perú, 11001 \\ E-mail: aquiles.bendezu@unica.edu.pe \\ Cynthia V. Bendezú Hernández \\ Ingeniero Químico, Ica, Perú \\ Universidad Nacional San Luis Gonzaga de Ica, Urb. Las Dunas H-15, Ica, Perú, 11001 \\ E-mail: bendezucynthia@gmail.com
}

\begin{abstract}
In the country, among the fundamental environmental problems is the contamination of surface waters of rivers, as in the case of the Pisco River. The objective of the research work was to determine the physicochemical and microbiological indicators, for their use in the irrigation of vegetable and animal beverages under the regulation of the D.S. 004-2017-MINAM, category 3 (Pisco river category). Four samples were collected at the four sampling points in the months of June-September of the year 2019, during the dry season, these samples were collected according to the methodology of the National Protocol for monitoring the quality of surface water resources. woolen. Parameters were measured in the field such as: $\mathrm{T}, \mathrm{pH}$, conductivity, total dissolved solids, $\%$ sodium chloride and turbidity, using a $\mathrm{pH}$ meter, multiparametric and turbidimeter. The concentration of the other physicochemical parameters and the microbiological parameters were determined in the UNSLG laboratories. The results were compared with D.S. 004-2017-MINAM. The parameters that were measured in the field comply with the ECA for surface waters of a category 3 river, while the physicochemical parameters such as: $\mathrm{Al}, \mathrm{Cu}, \mathrm{Fe}$ and $\mathrm{Zn}$ have higher values than the ECAs in all the sampling points. The microbiological parameters (Thermotolerant Coliforms and Escherichia coli) did not comply with the RCTs at points RP1 and RP2.The presence of these contaminants makes the water unsuitable for the purposes studied, in the same way for human consumption.
\end{abstract}

Keywords: Physicochemical, Microbiological Indicators, Pisco River

\section{RESUMEN}

En el país, entre los problemas ambientales fundamentales está la contaminación de las aguas superficiales de los ríos, como es el caso del río Pisco. El objetivo del trabajo de investigación fue determinar los indicadores fisicoquímicos y microbiológicos, para su uso en el riego de bebidas vegetales y animales bajo la norma del D.S. 004-2017-MINAM, categoría 3 (categoría río Pisco). Se tomaron cuatro muestras en los cuatro puntos de muestreo en los meses de junio-septiembre del año 2019, durante la época seca, estas muestras se tomaron de acuerdo a la metodología del Protocolo Nacional de monitoreo de la calidad de los recursos hídricos superficiales. lana. Se midieron en campo parámetros como: T, pH, conductividad, sólidos totales disueltos, $\%$ de cloruro de sodio y turbidez, utilizando un medidor de $\mathrm{pH}$, multiparamétrico 
y turbidímetro. La concentración de los demás parámetros físicoquímicos y los parámetros microbiológicos se determinaron en los laboratorios de la UNSLG. Los resultados se compararon con el D.S. 004-2017-MINAM. Los parámetros que se midieron en campo cumplen con el ECA para aguas superficiales de un río de categoría 3, mientras que los parámetros fisicoquímicos como: $\mathrm{Al}, \mathrm{Cu}, \mathrm{Fe}$ y $\mathrm{Zn}$ presentan valores superiores a los ECA en todos los puntos de muestreo. Los parámetros microbiológicos (Coliformes Termotolerantes y Escherichia coli) no cumplieron con los ECAs en los puntos RP1 y RP2.La presencia de estos contaminantes hace que el agua no sea apta para los fines estudiados, de igual manera para el consumo humano.

Palabras clave: Fisicoquímica, Indicadores Microbiológicos, Río Pisco.

\section{INTRODUCCIÓN}

Actualmente la contaminación de agua superficial es un problema grande, en vista de que actividades realizadas a nivel de industria, proyectos, empresas, actividades diarias no se cumple con la normatividad vigente. Es necesario realizar investigaciones que permitan generar conocimientos que contribuyan a determinar decisiones para optimizar los procedimientos productivos en cada sector evitando en todo momento el impacto al ambiente y las comunidades que se ubican en las zonas de influencia (Gonzales, G., Zevallos, A., Gonzales, C., Nunez, D., Gastanaga, C., Cabezas, C., Steenland, K., 2014).

Las causas principales de la contaminación de las aguas superficiales en América Latina posiblemente tendrían influencia el crecimiento poblacional, desarrollo de las actividades antrópicas como la agricultura, ganadería y el vertimiento de aguas negras sin tratamiento y residuos sólidos. Es esencial beber agua de buena calidad para la salud humana porque permite reducir muchas enfermedades (Rojas, 2016).

Estudio realizado por la ANA en Perú, en 129 cuencas hidrográficas se determinó que las quebradas y los ríos analizados están contaminados, con parámetros microbiológicos y metales pesados. La perturbación de la calidad del agua para el consumo humano y para actividades agropecuarias e industriales es a causa de las descargas de aguas grises y residuos sólidos que votan en las fuentes de agua en la cabecera de las cuencas (ANA, 2016).

Se justifica plenamente este proyecto de investigación por los aspectos ambientales de vigilancia y conservación de los ecosistemas fluviales, por las normas legales y la importancia de cumplir con la ley de los recursos hídricos que indican tácticamente toda actividad comercial e industrial tiene que tener en consideración el impacto sobre el medio ambiente, tratando en lo posible de evitar los riesgos de ruptura del equilibrio de nuestro ecosistema.

En la provincia de Pisco el agua del rio, se puede considerar que se esta contaminando por efluentes domesticos y mineros, aletrando la calidad del agua, su naturaleza y los productos hidrologicos, 
impidiendo su aprovechamiento. Por tal motivo la presente investigacion permite conocer la calidad fisicoquímica y microbiológica del agua del río Pisco, con el fin de informar a la poblacion de la problemática actual y buscar implementar actividades que sirvan para mitigar la contaminacion.

\section{OBJETIVOS}

Determinación de los parámetros físicoquímicos y microbiológicos del río Pisco

Contribuir a la identificación y caracterización de tecnologías de fácil implementación en regiones rurales de escaso desarrollo socioeconómico, que permita mantener y mejorar la calidad del agua para consumo humano.

\section{METODOLOGÍA}

Materiales utilizados en la investigación. - Para llevar a cabo el proyecto se realizaron ensayos experimentales, en los laboratorios de la Universidad Nacional "San Luis Gonzaga", Laboratorio de la Facultad de Ingeniería Ambiental y Sanitaria y el Laboratorio de la Facultad de Ciencias Biológicas. Para obtener los resultados se utilizaron equipos bien calibrados y confiables.

Población y Muestra. - La población esta conformada por las aguas de la cuenca del río Pisco e Ica. Se tomarán 04 muestras durante los cuatro meses (junio-setiembre 2019) con cuatro puntos de monitoreo de acuerdo a la metodología del Protocolo Nacional para el Monitoreo de la calidad de los recursos hídricos superficiales de la Autoridad Nacional del Agua (ANA, 2016)

La determinación se realizó durante los meses de junio-setiembre 2019, se tomaron como referencia de ubicación cuatro puntos de muestreo:

RP1: Puente Murga (Bernales), coordenadas UTM: 1343'16.4" S 7559'0.05"W

RP2: Frente al distrito de Independencia, coordenadas UTM: $13^{\circ} 42^{\prime} 46.6^{\prime \prime} \mathrm{S} 76^{\circ} 01^{\prime} 50.01^{\prime \prime} \mathrm{W}$

RP3: Frente al centro poblado de la Cuchilla, coordenadas UTM: 134'ㄴ․ $246^{\prime \prime} \mathrm{S} 76^{\circ} 03^{\prime} 36.6^{\prime \prime} \mathrm{W}$

RP4: Puente Huamani Panamericana Antigua, coordenadas UTM: 1341'14.4" S 7609'29.1"W

\section{Metodología de Análisis:}

1. Variación espacial y temporal de la calidad del agua del Río Pisco

Metodología de Análisis Fisicoquímicos: (a) Método potenciométrico (medición del pH), (b) Método nefelométrico (medición de la turbidez), (c) Método Conductimétrico (medición de la conductividad, $\mathrm{STD}, \%$ de $\mathrm{NaCl}$ ), (d) Método colorimétrico (medición de parámetros fisicoquímicos, $\mathrm{NO}_{3}{ }^{-}, \mathrm{NO}_{2}{ }^{-}, \mathrm{PO}_{3} \equiv$, $\mathrm{Al}, \mathrm{Cu}, \mathrm{Fe}$, etc., 
Metodología de Análisis Microbiológicos: Etapa de la investigación en la que se procedió a realizar el análisis bacteriológico de las muestras de agua a través de los tubos múltiples de fermentación, la cual consistió en determinar la densidad del grupo coliformes expresadas en términos del "Numero Más Probable" (NMP). Para lo cual se efectuó en 2 fases: Presuntiva, confirmativa (APHA, AWWA, WPCF, 1992)

2. Cálculo del índice de calidad del agua

Índice aritmético ponderado de la calidad del agua (WAWQI):

$$
W A W Q I=\frac{\sum_{i=1}^{i=n} Q_{i} W_{i}}{\sum W_{i}} \quad Q_{I}=\frac{V_{I}-V_{d I}}{S_{I}-V_{d I}} \quad k=\frac{1}{\sum 1 / S_{I}}
$$

where

$W_{i}=$ unit weight for each water quality parameter;

$K=$ proportionality constant;

$Q_{i}=$ the quality rating scale for each parameter;

$Q_{\text {actual }}=$ estimated concentration of $i$ th parameter in the analyze

$Q_{\text {ideal }}=$ the ideal value of this parameter in pure water,

$S_{\text {standard }}=$ recommended standard value of $i$ th parameter;

$n=$ number of water quality parameters.

3. Propuesta de acciones de mejora

Se propone diversos tratamientos de acuerdo a los resultados obtenidos

\section{RESULTADOS Y DISCUSIÓN}

Variación promedio de cada Parámetro Físico en los Puntos de Muestreo del Río Pisco:

En las figuras 1 y 2 se observa las variaciones determinadas resaltantes en los cuatro puntos de muestreo en los meses de junio-setiembre del año 2019, en la conductividad, la turbidez y los STD son mayores en el punto RP1; la temperatura se mantiene; el pH y el \% de $\mathrm{NaCl}$ se mantienen constante. 
Figura 1: Valores Promedio de cada Parámetro Físico (conductividad y STD) en los Cuatro Puntos de Muestreo de la Cuenca del Rio Pisco

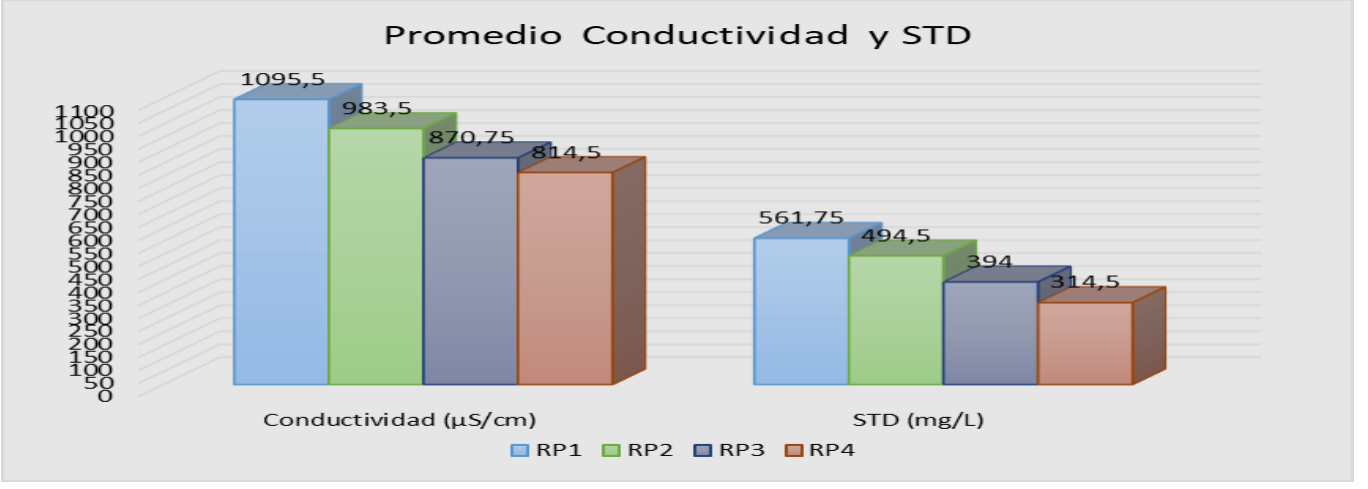

Figura 2: Valores Promedio de cada Parámetro Físico (T, pH, \% NaCl y Turbidez) en los Cuatro Puntos de Muestreo de la Cuenca del Rio Pisco

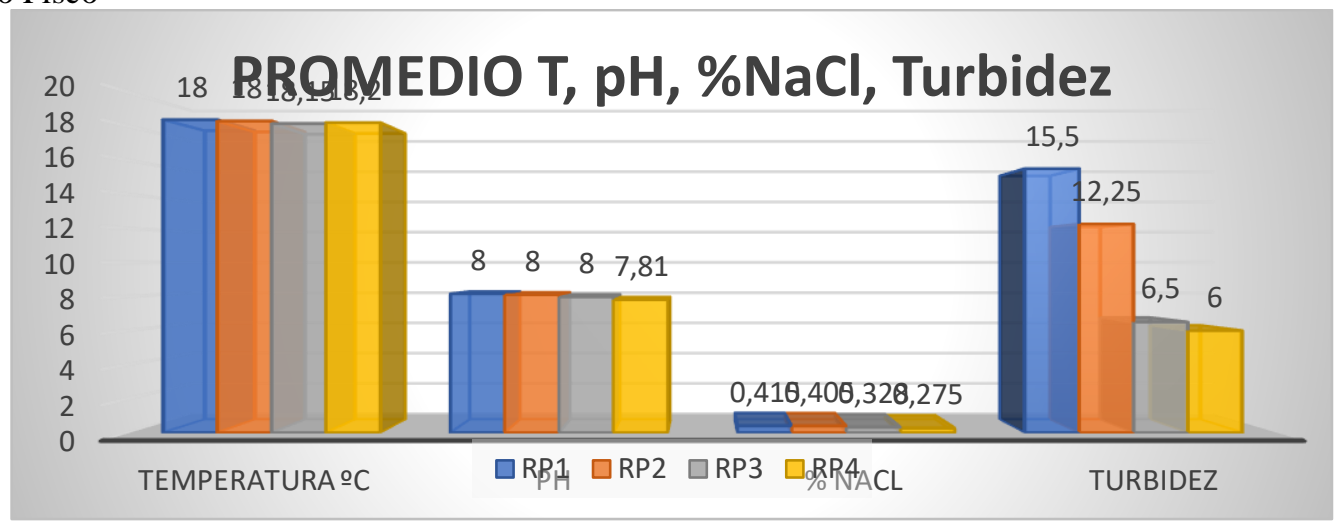

Variación promedio de cada Parámetro Químico en los Puntos de Muestreo del Río Pisco

En la figura 3 se observa las variaciones determinadas en los cuatro puntos de muestreo, las diferencias que más resaltan son en loa nitratos y los nitritos es mayor en RP1 y aguas abajo va disminuyendo; en los demás parámetros evaluados se mantiene aproximadamente constante.

Figura 3: Valores Promedio de cada Parámetro Químico en los Cuatro Puntos de Muestreo de la Cuenca del Río Pisco

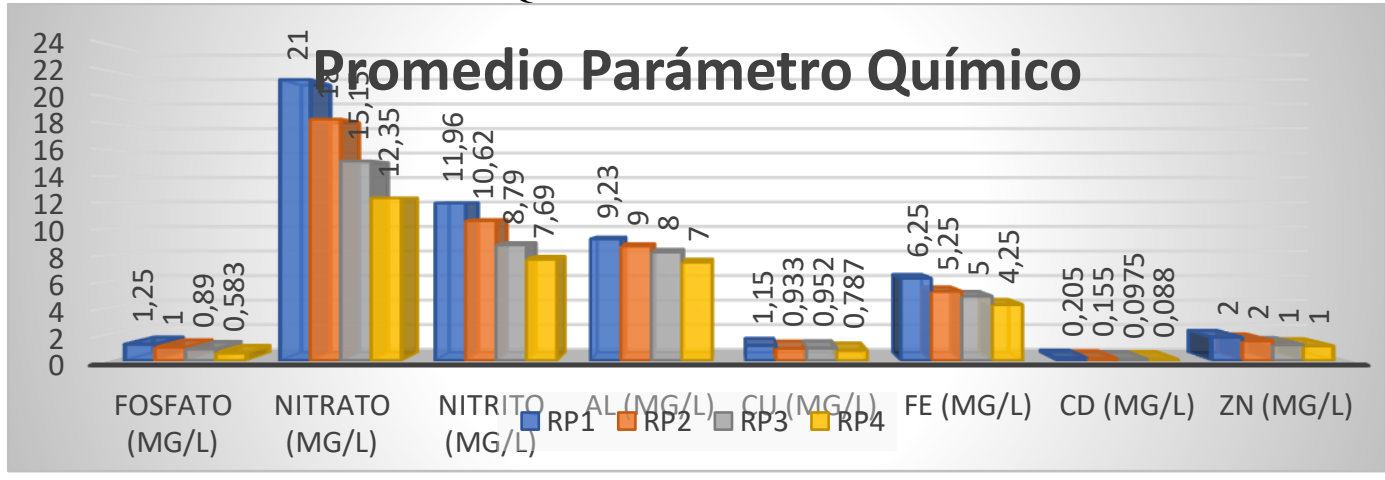

Variación promedio de cada Parámetro Microbiológico de los Puntos de muestreo del Río Pisco

En la figura 4 se observa en los coliformes Termotolerantes y Escherichia coli en los puntos RP1 y RP2 son mayores y aguas abajo va disminuyendo. 
Figura 4: Valores Promedio de cada Parámetro microbiológico en los Cuatro Puntos de Muestreo de la Cuenca del Río Pisco PROMEDIO

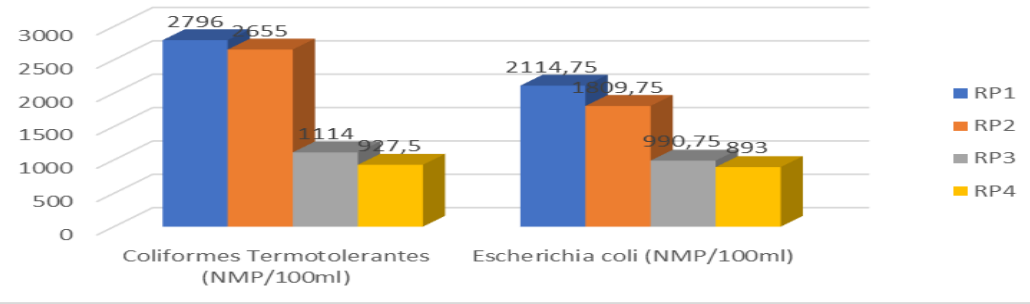

Cálculo del índice de calidad del agua

Cálculo del WAWQI en el punto RP1: Dado que es punto que tiene valores más elevados en cuánto a los parámetros fisicoquímicos comparados con nuestra normativa peruana. Se decidió determinar el WAWQI en el RP1como se muestra en la tabla 1.

Tabla 1: Cálculo del WAWQI en el punto RP1

\begin{tabular}{|c|c|c|c|c|c|c|c|c|c|}
\hline Parámetro & Unidad & $\mathbf{V}_{\mathbf{I}}$ & $S_{I}$ & $V_{\text {dI }}$ & $\mathbf{Q}_{\mathbf{I}}$ & $1 / S_{I}$ & $\mathbf{K}$ & $\mathbf{W}_{\mathbf{I}}$ & $\mathbf{Q}_{\mathbf{I}} * \mathbf{W}_{\mathrm{I}}$ \\
\hline pH & $\begin{array}{l}\text { unidad } \\
\text { de pH }\end{array}$ & 8.155 & 8.5 & 7 & 77.00 & 0.12 & 0.885 & 0.1041 & 8.02 \\
\hline conductividad & $(\mu \mathrm{S} / \mathrm{cm})$ & 1095.5 & 2500 & $\mathbf{0}$ & 43.82 & 0.0004 & 0.885 & 0.0004 & 0.02 \\
\hline Nitrato & $(\mathrm{mg} / \mathrm{L})$ & 21.325 & 100 & $\mathbf{0}$ & 21.33 & 0.01 & 0.885 & 0.0089 & 0.19 \\
\hline Nitrito & $(\mathrm{mg} / \mathrm{L})$ & 11.960 & 10 & $\mathbf{0}$ & 119.60 & 0.10 & 0.885 & 0.0885 & 10.58 \\
\hline Al & (mg/L) & 9.230 & 5 & $\mathbf{0}$ & 184.60 & 0.20 & 0.885 & 0.1770 & 32.67 \\
\hline $\mathbf{F e}$ & (mg/L) & 6.250 & 5 & $\mathbf{0}$ & 125.00 & 0.20 & 0.885 & 0.1770 & 22.13 \\
\hline Zn & (mg/L) & 1.775 & 2 & $\mathbf{0}$ & 88.75 & 0.50 & 0.885 & 0.4425 & 39.27 \\
\hline & & & & & & 1.13 & & 1.00 & 112.88 \\
\hline
\end{tabular}

Fuente: Elaboración propia del autor

Cálculo del WAWQI en el punto RP4: Dado que es punto que tiene valores más bajos de los puntos muestreados en cuánto a los parámetros fisicoquímicos. Se decidió determinar el WAWQI en el RP4como se muestra en la tabla 2.

Tabla 2: Cálculo del WAWQI en el punto RP4

\begin{tabular}{|c|c|c|c|c|c|c|c|c|c|}
\hline Parámetro & Unidad & $\mathbf{V}_{\text {I }}$ & $\mathbf{S}_{\text {I }}$ & $\mathbf{V}_{\text {dI }}$ & $\mathbf{Q}_{I}$ & $1 / S_{I}$ & $\mathbf{K}$ & $\mathbf{W}_{\text {I }}$ & $\mathbf{Q}_{\mathbf{I}} * \mathbf{W}_{\mathbf{I}}$ \\
\hline pH & $\begin{array}{l}\text { unidad } \\
\text { de } p H\end{array}$ & 7.81 & 8.5 & 7 & 54.00 & 0.12 & 0.885 & 0.1041 & 5.6224 \\
\hline conductividad & $(\mu \mathrm{s} / \mathrm{cm})$ & 814.50 & 2500 & $\mathbf{0}$ & 32.58 & 0.0004 & 0.885 & 0.0004 & 0.0115 \\
\hline Nitrato & (mg/L) & 12.35 & 100 & $\mathbf{0}$ & 12.35 & 0.01 & 0.885 & 0.0089 & 0.1093 \\
\hline
\end{tabular}




\begin{tabular}{|l|l|r|r|r|r|r|r|r|r|}
\hline Nitrito & $(\mathrm{mg} / \mathrm{L})$ & 7.69 & 10 & 0 & 76.90 & 0.10 & 0.885 & 0.0885 & 6.8057 \\
\hline Al & $(\mathrm{mg} / \mathrm{L})$ & 7.47 & 5 & 0 & 149.30 & 0.20 & 0.885 & 0.1770 & 26.4261 \\
\hline Fe & $(\mathrm{mg} / \mathrm{L})$ & 4.25 & 5 & 0 & 85.00 & 0.20 & 0.885 & 0.1770 & 15.0450 \\
\hline Zn & $(\mathrm{mg} / \mathrm{L})$ & 1.12 & 2 & 0 & 56.10 & 0.50 & 0.885 & $\mathbf{0 . 4 4 2 5}$ & $\mathbf{2 4 . 8 2 4 3}$ \\
\hline & & & & & & 1.13 & & 1.00 & 78.844 \\
\hline
\end{tabular}

Fuente: Elaboración propia del autor

Para la clasificación de la calidad del agua utilizando el índice aritmético ponderado, se utilizó la tabla 3 .

Tabla 3 . Clasificación de la calidad del aqua para consumo humano utilizando el índice aritmético ponderado de la calidad del agua

\begin{tabular}{cc}
\hline WAWQI & $\begin{array}{c}\text { Calidad del agua } \\
\text { Clasificación }\end{array}$ \\
\hline$<25$ & Excelente \\
\hline $26-50$ & Bien \\
\hline $51-75$ & Malo \\
\hline $76-100$ & Muy mal \\
\hline$>100$ & Inadecuado para beber \\
\hline
\end{tabular}

Fuente: Brown, R. M. et al. 1972. A Water Quality Index Crashing the

Physiological Barrier. Indic Environ Qual, 1, 173-182

\section{Discusión:}

En el presente trabajo de investigación de acuerdo a los datos obtenidos como la conductividad, los sólidos totales disueltos, nitratos y nitritos son más elevados aguas arriba es decir el punto de muestreo RP1, aguas abajo va disminuyendo; en el caso del pH, T, y demás parámetros fisicoquímicos se mantiene con poca variación. En el caso de los parámetros microbiológicos es preocupante porque tiene valores elevados en los puntos RP1y RP2 es debido que las aguas residuales domesticas de la población del distrito de Humay son directamente vertidos al cuerpo de las aguas superficiales del Río Pisco. El Río Pisco está dentro de la categoría 3, clase 3, el código de la cuenca es 13752 y la identificación del cuerpo de agua es13752. Según el resultado de nuestros datos la mayoría están dentro de los ECAs, con excepción del Al, $\mathrm{Cu}, \mathrm{Fe}, \mathrm{Zn}$, que se encuentran por encima de los valores de los ECAs. De igual manera los parámetros microbiológicos como los coliformes Termotolerantes y Escherichia coli están por encima de los ECAs en los puntos RP1 y RP2. 


\section{CONCLUSIONES}

1. Los parámetros fisicoquímicos y microbiológicos de las muestras no difieren significativamente en los meses de junio-setiembre del 2019 en los diferentes puntos de muestreo.

2. Los parámetros físicos se encuentran dentro de valores permitidos por el ECA

3. Los parámetros químicos como el $\mathrm{Al}, \mathrm{Cu}, \mathrm{Fe}, \mathrm{Zn}$ son ligeramente superiores a los establecidos por el ECA.

4. Los parámetros microbiológicos como coliformes Termotolerantes y Escherichia coli en los puntos RP1 y RP2 están por encima de los límites permitidos por el ECA.

5. El WAWQI en el punto RP1 fue de 112.8 que clasifica el agua como inadecuada para beber y el que WAWQI en el punto RP4 fue de 78.8 que clasifica el agua como de mala calidad

6. Para las propuestas de tratamiento tener en cuenta la especiación de algunos metales, como equipo de trabajo sugerimos las siguientes propuestas Osmosis inversa, adsorción-precipitación, intercambio iónico, nanofiltración, tratamiento biológico; de acuerdo al presupuesto y al destino final del agua. 


\section{REFERENCIAS BIBLIOGRÁFICAS}

1. Álvarez, C., Vergara, F., Acevedo, R., \& Severiche, C. (2014). Evaluación Analítica para la Determinación de Arsénico y Selenio en Aguas por Espectroscopía de Absorción Atómica.

2. ANA. (2016). Protocolo Nacional para el Monitoreo de la Calidad de los Recursos Hídricos Superficiales.

3. Ausejo, F. (2010). Propuesta de Investigación Caos y Orden en la Gestión del Agua en el Perú. Lima: Tesis Doctoral PUCP.

4. Bueno, J. (1997). Contaminación e Ingeniería Ambiental. Oviedo España.

5. De vida. (2012). Estudio de la Calidad de Agua en el Valle del Rio Apurímac, Contando con el apoyo financiero de USAID y del gobierno peruano. Perú.

6. Diaz, P. (2018). Determinación de la Calidad Fisicoquímica y Microbiológica del Agua de la Quebrada Chupishiña, Distrito de Rumisapa, Provincia de Lamas y Región San Martin. Repositorio de Tesis, Universidad Peruana Unión.

7. Guevara, A. (1996). Métodos de Análisis para la Evaluación de la Calidad del Agua CEPIS/OPS/OMS. Lima Perú.

8. Leiva, D., Chávez, J. y Corroto, F. (2014). Evaluación de la Calidad Fisicoquímica y Microbiológica del río Shocol, provincia de Rodríguez de Mendoza, Amazonas.

9. Ministerio de salud, (2007). Dirección General de Salud Ambiental. "DIGESA". Protocolo de Monitoreo de la Calidad Sanitaria de los Recursos Hídricos Superficiales. Lima Perú.

10. Organismo Mundial de la Salud, (2015). Agua.

11. Reyes, C. (2012). Estudio de la Contaminación de las Aguas del Rio Chillón. Lima-Perú.

12. Rojas, M. (2011). Estudios de la Contaminación de los Recursos Hídricos en la Cuenca del Rio San Pedro, Previos a la Construcción de una Hidroeléctrica. México.

13. APHA, AWWA, WPCF. (1992). Métodos Normalizados para el Análisis de aguas potables y residuales. Madrid- España

14. Gonzales, G., Zevallos, A., Gonzales, G., Núñez, D., Gastanaga, C., Cabezas, C., Steeland, K. (2014). Contaminación Ambiental Variabilidad Climática y Cambio Climático. Una revisión del Impacto en la Salud de la Población Peruana. 